\title{
A Control Strategy with Tactile Perception Feedback for EMG Prosthetic Hand
}

\author{
Changcheng Wu, Aiguo Song, Yun Ling, Nan Wang, and Lei Tian \\ School of Instrument Science and Engineering, Southeast University, Nanjing 210096, China \\ Correspondence should be addressed to Aiguo Song; a.g.song@seu.edu.cn
}

Received 6 September 2014; Revised 13 January 2015; Accepted 18 January 2015

Academic Editor: Lei Wang

Copyright (C) 2015 Changcheng Wu et al. This is an open access article distributed under the Creative Commons Attribution License, which permits unrestricted use, distribution, and reproduction in any medium, provided the original work is properly cited.

\begin{abstract}
To improve the control effectiveness and make the prosthetic hand not only controllable but also perceivable, an EMG prosthetic hand control strategy was proposed in this paper. The control strategy consists of EMG self-learning motion recognition, backstepping controller with stiffness fuzzy observation, and force tactile representation. EMG self-learning motion recognition is used to reduce the influence on EMG signals caused by the uncertainty of the contacting position of the EMG sensors. Backstepping controller with stiffness fuzzy observation is used to realize the position control and grasp force control. Velocity proportional control in free space and grasp force tracking control in restricted space can be realized by the same controller. The force tactile representation helps the user perceive the states of the prosthetic hand. Several experiments were implemented to verify the effect of the proposed control strategy. The results indicate that the proposed strategy has effectiveness. During the experiments, the comments of the participants show that the proposed strategy is a better choice for amputees because of the improved controllability and perceptibility.
\end{abstract}

\section{Introduction}

Prosthetic hands are of great importance to the upper limbs amputees which can help them to complete some manipulations such as grasping. How to make the prosthetic hand grasp objects by following inclinations of amputees is a meaningful research direction. Scholars in this filed have done a lot of research work and made some important development.

At present, there are some kinds of prosthetic hands: decorative prosthetic hand (for the purpose of decoration, without function), switches controlled prosthetic hand, electromyography (EMG) signal controlled prosthetic hand, and so forth. A typical control scheme of EMG prosthetic hand is shown in Figure 1 [1]. EMG 1 and $\mathrm{EMG}_{2}$ are EMG signals acquired from a pair of antagonistic muscles, $K_{E}$ is the scale factor, $F_{d}$ is the expected grasp force, and $F_{n}$ is the grasp force measured by force sensors. $K_{n}$ is the feedback gain and $K_{p}$ is the proportional gain for the force error. This control mode is popular due to its simple operation and in accordance with the operation habits of natural hand. However, there are still some problems to be solved. Firstly, this control mode does not fully consider the influence of EMG signals on control strategy: the EMG signals measured from different persons are different, and the EMG signals measured from different state of the same individual may be different. Secondly, this control mode lacks perceptibility: the user cannot perceive the grasp force of the prosthetic hand when grasping an object.

EMG motion pattern recognition is a basic technique of EMG prosthetic hand; a great number of recognition methods have been developed, such as time-domain method and frequency-domain method [2]. Parameters of ARMA model and Kalman filter were adopted as character vectors to identify the movement patterns [3]. Khoshaba et al. used the integral absolute value of the EMG signals to recognize the movement patterns [4]. Autoregressive (AR) model, power spectrum, wavelet coefficients, neural network, and some signal processing methods were also used [5]. However, due to the difference of the sticking positions of EMG sensors and the difference of temperature and humidity of the environment, the EMG signals measured from different persons or different states of the same individual may lead to different results. This kind of phenomenon may affect 


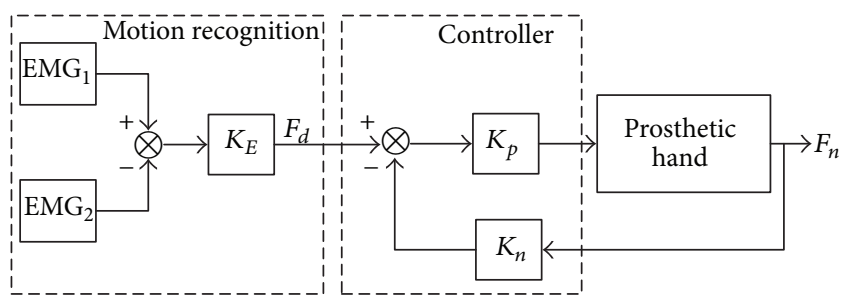

FIGURE 1: A typical control scheme of the EMG prosthetic hand.

the accuracy of the movement pattern recognition, which may cause descending of the control effect.

The design of the controller is another important part of the EMG prosthetic hand; most of the control strategies for the prosthetic hands aimed at the controllability, such as accuracy force tracking control and speed control of opening and closing. In order to get a satisfying grasping performance, Fassih et al. proposed a control strategy based on defining virtual spring-damper model between two finger tips and damping force at each finger joint [6]. Chen et al. focused on hybrid of soft computing technique of adaptive neuron-fuzzy inference system (ANFIS) and hard computing technique of adaptive control for a two-dimensional movement with thumb and index prosthetic hand [7]. A hybrid sliding modebackstepping (HSMBS) parallel force-velocity controller was proposed to improve the control effect of powered prosthetic hands by Engeberg and Meek [8]. According to the tactile feedback and visual feedback, when grasping an object, natural hand can adjust the grasp force in time. But for prosthetic hand which has no tactile feedback to the user, it is hard to obtain expected control effect. So a prosthetic hand which is not only controllable but also appreciable is probably a good choice for amputees. The controllability of the prosthetic hand just functionally assists amputees to complete some simple actions such as grasping. However, the perceptibility of the prosthetic hand considers more about the amputee himself. It not only satisfies the functional needs of the hands but also meets the psychological requirements. In a view of the perceptibility of the prosthetic hand, amputees get more information when using it. It shows a faster acceptance for amputees when using the hand for a long time, and this phantom limb feeling may also improve the control effect.

This paper describes a control strategy for the EMG prosthetic hand, which mainly includes EMG self-learning recognition, backstepping controller with stiffness fuzzy observation, and grasp force tactile representation. EMG selflearning recognition aims to reduce the influence on EMG signals caused by the uncertainty of the contacting positions of the EMG sensors. Backstepping controller with stiffness fuzzy observation is used to realize the position control and grasp force control. The grasp force tactile representation aims to improve the proprioception of the prosthetic hand, which can improve the control effect. Finally, experiments were implemented to verify the proposed control strategy.

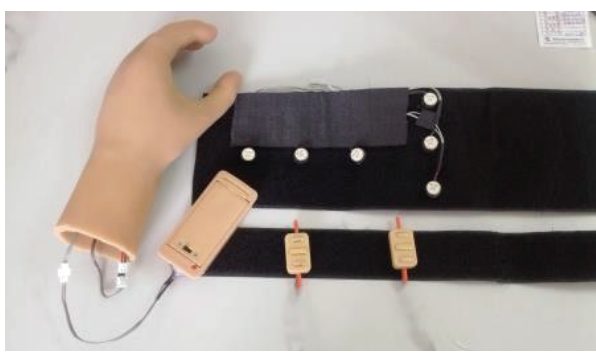

FIGURE 2: MPH-III prosthetic hand.

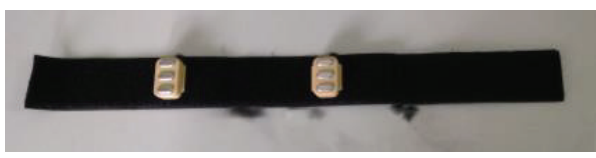

FIGURE 3: EMGs acquisition device.

\section{System Components}

The MPH-III prosthetic hand, which is designed by Robot Sensor and Control Lab in Southeast University, is used in this paper. The MPH-III consists of three components: prosthetic hand, EMGs acquisition device, and tactile representation device (see Figure 2). The lithium batteries are used as power source for all components.

2.1. Prosthetic Hand. The MPH-III is a one-DOF (degree of freedom) prosthetic hand, which is equipped with three force sensors (to measure the grasp force) and a position sensor (encoder). Wearing silicone glove, the MPH-III looks like a natural hand. The core of the control system is a single chip microcomputer (C8051F320, Silicon Laboratories) [9]. The control board has a USB interface on which data can be exchanged between prosthetic hand's control board and computer.

2.2. EMGs Acquisition Device. Two EMG sensors are adopted to acquire EMG signals. A 10-bit A/D converter is used to digitize signals (sampling ratio is $1 \mathrm{kHz}$ ). A Bluetooth module is utilized to transmit the EMG signals to the controller of prosthetic hand. All the components of the EMGs acquisition device are fixed on a ribbon (see Figure 3) to make it convenient for users to wear, and the positions of the EMG sensors on the ribbon are adjustable because the detecting positions for different users are different.

2.3. Tactile Representation Device. The tactile representation device (TRD) consists of six vibration motors, which are controlled by an electronic system equipped with a Bluetooth module. The vibration motors are fixed on a ribbon (see Figure 4). When the ribbon is worn on the upper arm, the distribution of vibration motors is shown in Figure 5. These vibration motors may generate stimulation on the skin of the amputee. The TRD receives the force information of the prosthetic hand from the control module via Bluetooth module. Then command is generated to control the vibration 


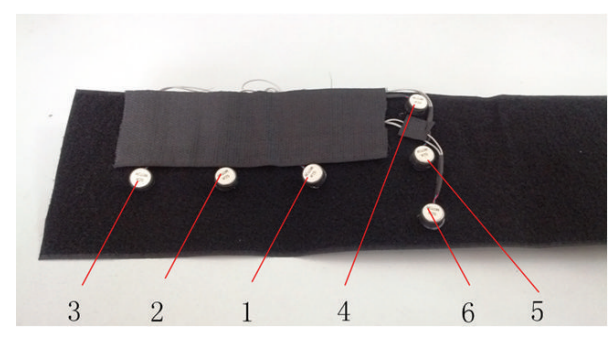

FIgURE 4: Tactile representation device.

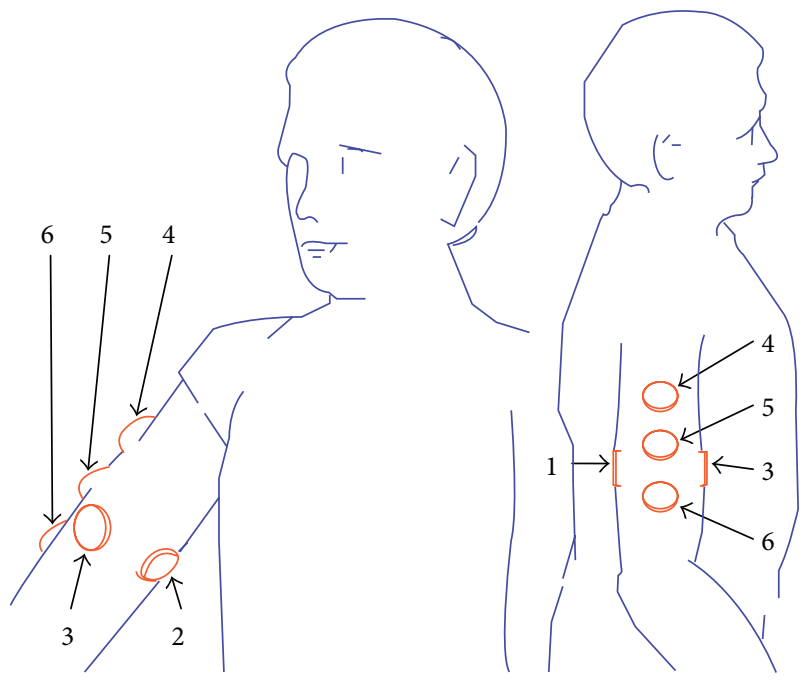

FIGURE 5: The distribution of the vibration motors.

motors orderly, so that the user can perceive the force states of the prosthetic hand.

\section{Design of the Control Strategy}

The designed control strategy is based on manipulation patterns of the natural hand. User plays a dominant role in the system. When she/he intends to operate the prosthetic hand, a control command will be generated from the brain and transmitted to motor nerves through spinal cord. The motor nerves control the muscle movement. And the EMG signal will be generated on the surface of skin at that time. These EMG signals can be used to control the prosthetic hand after processing. Processing these EMG signals probably involves amplifying, filtering, acquiring, feature extracting, and motion identifying. The force sensors installed in fingers of the prosthetic hand are used to detect the grasp force. On one hand, the grasp force information feeds back to the controller; on the other hand it feeds back to the user through a certain tactile feedback type. According to the specialty of the object, users can combine visual and tactile message and adjust the control strategy in time. In this way, three closed loops are achieved to control the prosthetic hand: the first one is from user to prosthetic hand then back to the user through tactile feedback device, the second one is from controller to prosthetic hand then back to the controller, and the last one is from the user to prosthetic hand then back to the user through user's eyes. The functional scheme of this control strategy is shown in the Figure 6.

3.1. EMG Self-Learning Recognition. The accuracy of the pattern recognition of EMG signals is directly related to the control effect of the prosthetic hand. The surface EMG signal can illustrate the activity of skeletal muscles, and its amplitude ranges from less than $50 \mu \mathrm{V}$ to $30 \mathrm{mV}$, and the frequency range is from dozens to hundreds $\mathrm{Hz}$, depending on the muscle under observed.

Because the EMG signal strengths of different users are different and due to some other factors, an EMG self-learning recognition method is proposed as shown in Figure 9. Before the pattern recognition, EMG signals are processed as shown in Figure 7.

EMG sensors are attached to the surface of the muscle to acquire the EMG signals. Figures 8(a), 8(b), and 8(c) show the amplified EMG signals, the shaped EMG signals, and the filtered EMG signals, respectively.

In Figure 9, $\mathrm{EMG}_{1}$ and $\mathrm{EMG}_{2}$ are the EMG signals which are preprocessed as shown in Figure 7. The values of $\mathrm{EMG}_{1}$ and $\mathrm{EMG}_{2}$ are between $0 \mathrm{~V}$ and $3.3 \mathrm{~V}$. The EMG learner is designed to record and update the minimum and maximum values of the EMG signals. A moving window is adopted, and the principles of the recording and updating are as follows:

Step 1. Calculate the average value (Ave) of the data in moving time window:

$$
\text { Ave }=\frac{1}{N} \sum_{n=0}^{N-1} \mathrm{EMG}_{1}(t-n)
$$

where $N$ is the length of the time window. $\mathrm{EMG}_{1}(t)$ represents the current data of the EMG signal and $\mathrm{EMG}_{1}(t-n)$ represents the previous $n$th data of the EMG signal.

Step 2. Update the data:

$$
\begin{aligned}
& \operatorname{Max}_{E 1}= \begin{cases}\operatorname{Max}_{E 1}, & \operatorname{Max}_{E 1} \geq \text { Ave } \\
\text { Ave, } & \operatorname{Max}_{E 1}<\text { Ave }\end{cases} \\
& \operatorname{Min}_{E 1}= \begin{cases}\operatorname{Min}_{E 1}, & \operatorname{Min}_{E 1} \leq \text { Ave } \\
\text { Ave, } & \operatorname{Min}_{E 1}>\text { Ave }\end{cases}
\end{aligned}
$$

where $\operatorname{Max}_{E 1}$ and $\operatorname{Min}_{E 1}$ are the maximum and minimum values of the $\mathrm{EMG}_{1}$, respectively. The initial value of $\mathrm{Max}_{E 1}$ is set to 0 , and the initial value of $\operatorname{Min}_{E 1}$ is set to 3.3.

The maximum and minimum values of the $\mathrm{EMG}_{2}, \mathrm{Max}_{\mathrm{E2}}$, and $\operatorname{Min}_{E 2}$ are updated by using the same method.

Adjustable factors $\left(K_{E 1}, K_{E 2}\right)$ are defined as follows:

$$
\begin{aligned}
K_{E 1} & =\frac{1}{\operatorname{Max}_{E 1}-\operatorname{Min}_{E 1}}, \\
K_{E 2} & =\frac{1}{\operatorname{Max}_{E 2}-\operatorname{Min}_{E 2}} .
\end{aligned}
$$




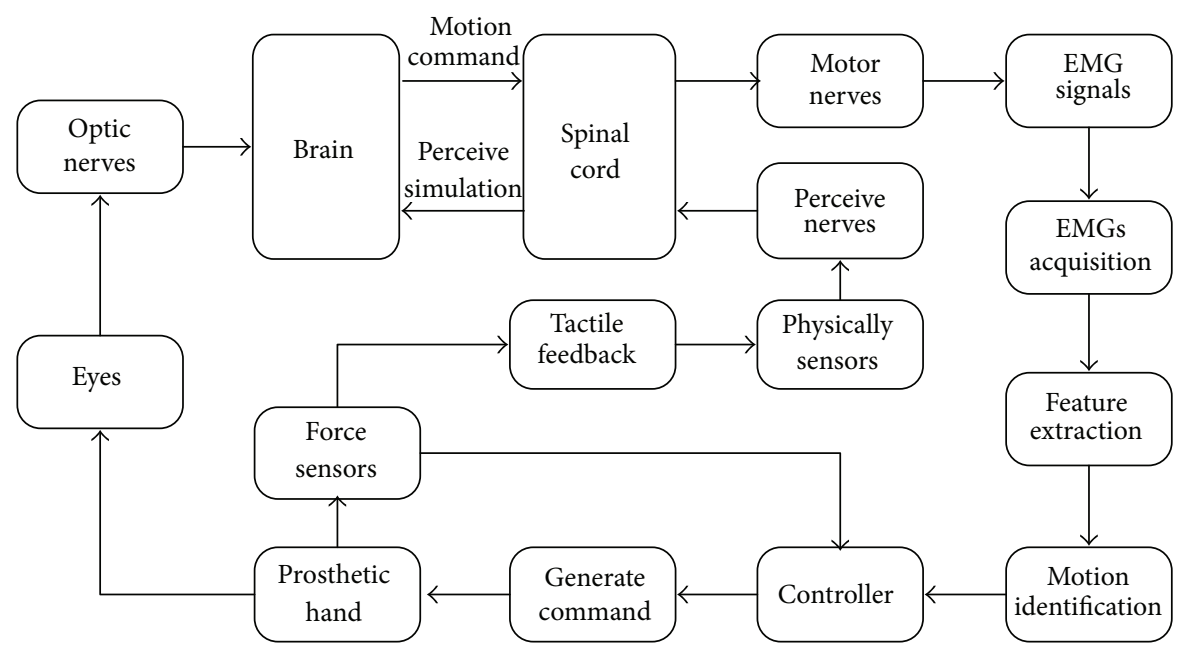

Figure 6: Control strategy functional scheme.

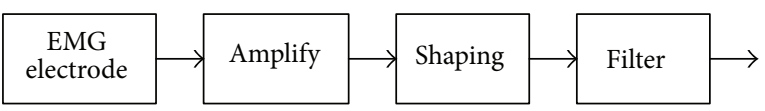

FIGURE 7: Preprocessing scheme of the EMG signals.

The output, $F_{d}$, is determined by $\mathrm{EMG}_{1}$ and $\mathrm{EMG}_{2}$, which is as follows:

$$
\begin{aligned}
F_{d}= & {\left[\left(\mathrm{EMG}_{1}-\mathrm{Min}_{E 1}\right) \times K_{E 1}-\left(\mathrm{EMG}_{2}-\mathrm{Min}_{E 2}\right) \times K_{E 2}\right] } \\
& \times K_{E}
\end{aligned}
$$

where $K_{E}$ is the scale factor.

In free space, $F_{d}$ reflects the closing or opening speed of the prosthetic hand, and in restricted space, $F_{d}$ reflects the grasp force.

With the help of this recognition method, the influence of the diversity of the EMG signals on the accuracy of the pattern recognition is reduced.

\subsection{Backstepping Controller with Stiffness Fuzzy Observation.} To realize the position control and grasp force control, a backstepping controller with stiffness fuzzy observation (BCSFO) is designed in this paper. The designed controller is shown in Figure 10. The input signal, $F_{d}$, is the output of the motion recognizer. $F_{n}$ is the grasp force measured by force sensor which is attached to the prosthetic hand's finger, $K_{n}$ is the scaling factor, $K_{n d}$ is the differential scaling factor, and $u$ is the voltage applied to motor. $x_{1}$ and $x_{2}$ are, respectively, the position and velocity of the prosthetic hand's finger. $k$ is object's stiffness and the stiffness is defined as

$$
k=\frac{F_{n}}{x_{0}-x_{1}}(N / \circ)
$$

where $x_{0}$ is the original size of the object and it is the position of the prosthetic hand's finger when the object and finger contact for the first time.

In free space, the output of the planner is as follows:

$$
\theta_{d}=\theta_{0}+\int\left(F_{d}-K_{n} F_{n}-K_{n d} \frac{d}{d t} F_{n}\right) d t=\theta_{0}+\int F_{d} d t
$$

where $\theta_{0}$ is the position when prosthetic hand's finger changes from restricted space to free space.

In restricted space, the stiffness of the object and the deformation of the structure may affect the relationship between the grasp force of the prosthetic hand and the angle of the motor rotation. Since the range of the designed grasp force is relatively small $(0 \sim 30 \mathrm{~N})$, the influence of the structure deformation is ignored, and the output of the planner in restricted is as follows:

$$
\theta_{d}=\theta_{n}+\frac{F_{d}-\left(K_{n} F_{n}+K_{n d}(d / d t) F_{n}\right)}{k} .
$$

The system model of prosthetic hand is selected as follows:

$$
\begin{gathered}
\dot{x}_{1}=x_{2}, \\
\dot{x_{2}}=m\left(x_{1}, x_{2}\right)+n u, \\
m\left(x_{1}, x_{2}\right)=-\frac{B}{J} x_{2}-\frac{D}{J},
\end{gathered}
$$

where $B, J$, and $D$ are, respectively, the inertia, damping, and unknown nonlinear damping of the system. $u$ is the output of the system, and it is the control voltage of the motor. 


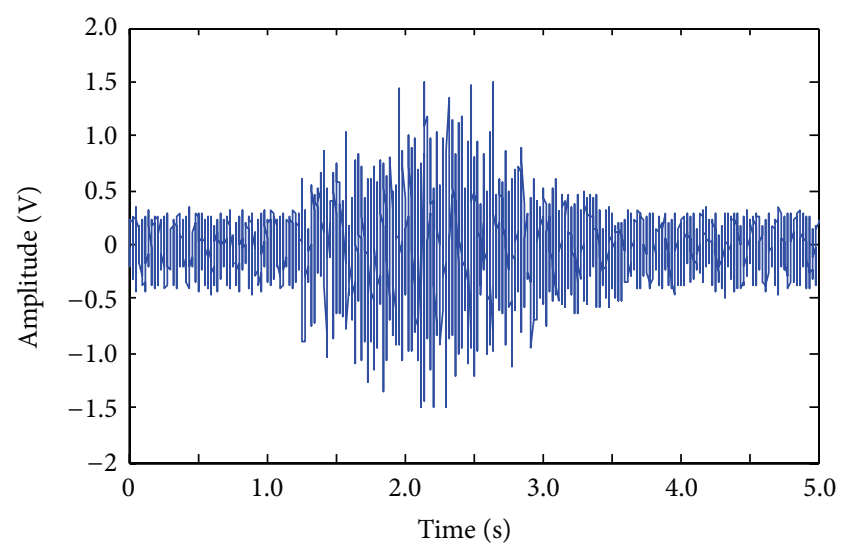

(a)

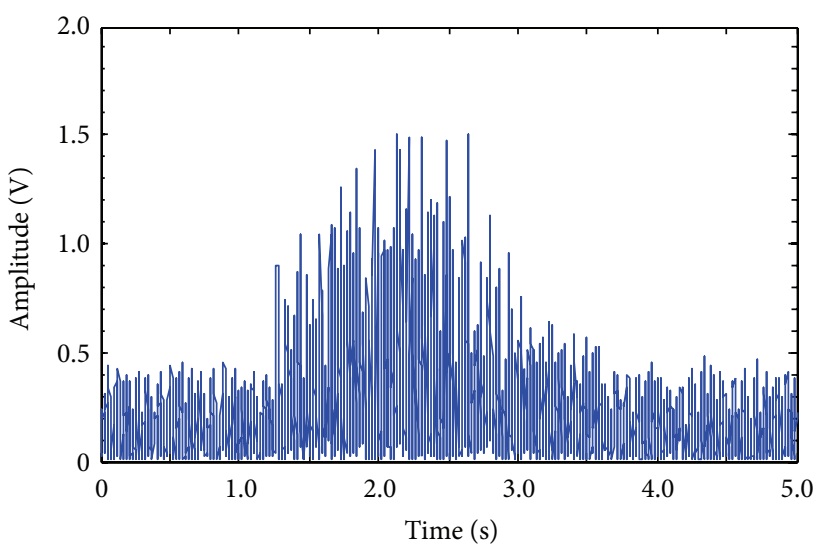

(b)

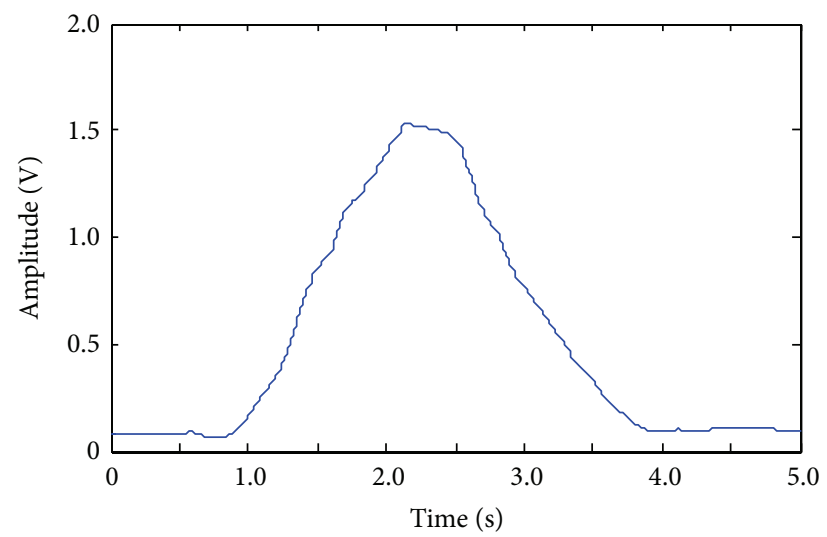

(c)

FIGURE 8: Amplified, shaped, and filtered EMG signals.

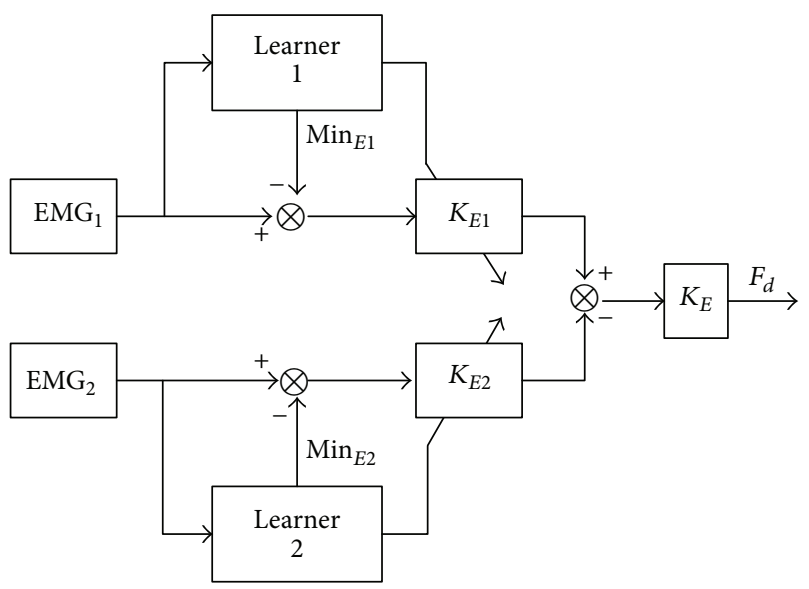

FIGURE 9: EMG signal self-learning recognition scheme.

Two error subsystems are defined as

$$
\begin{aligned}
& Z_{1}=x_{1}, \\
& Z_{2}=x_{2}-\alpha_{1}\left(x_{1}\right),
\end{aligned}
$$

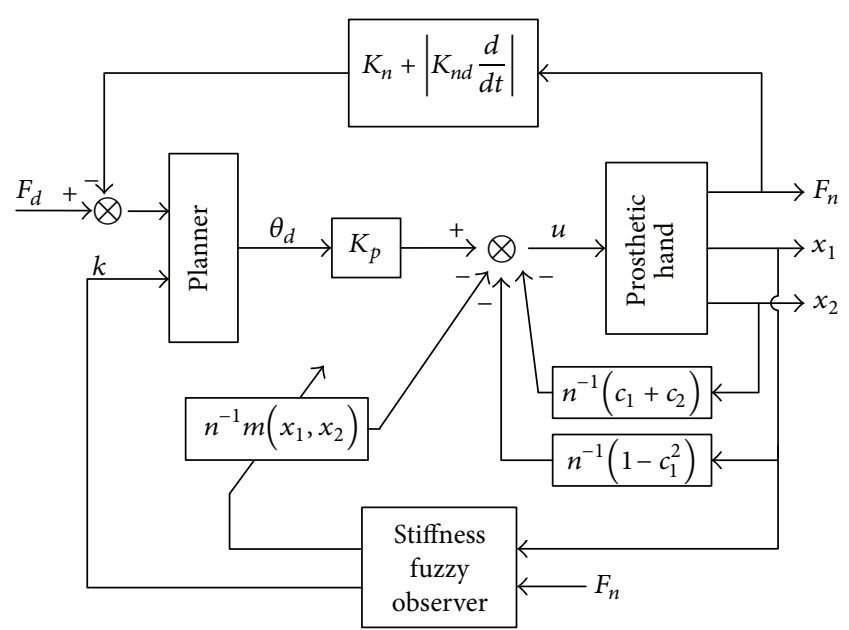

FIGURE 10: The block diagram of the backstepping controller with stiffness fuzzy observation.

where $\alpha_{1}\left(x_{1}\right)$ is a virtual control variable (i.e., the estimate of $\left.x_{2}\right)$ :

$$
\dot{Z}_{1}=\dot{x}_{1}=x_{2}
$$



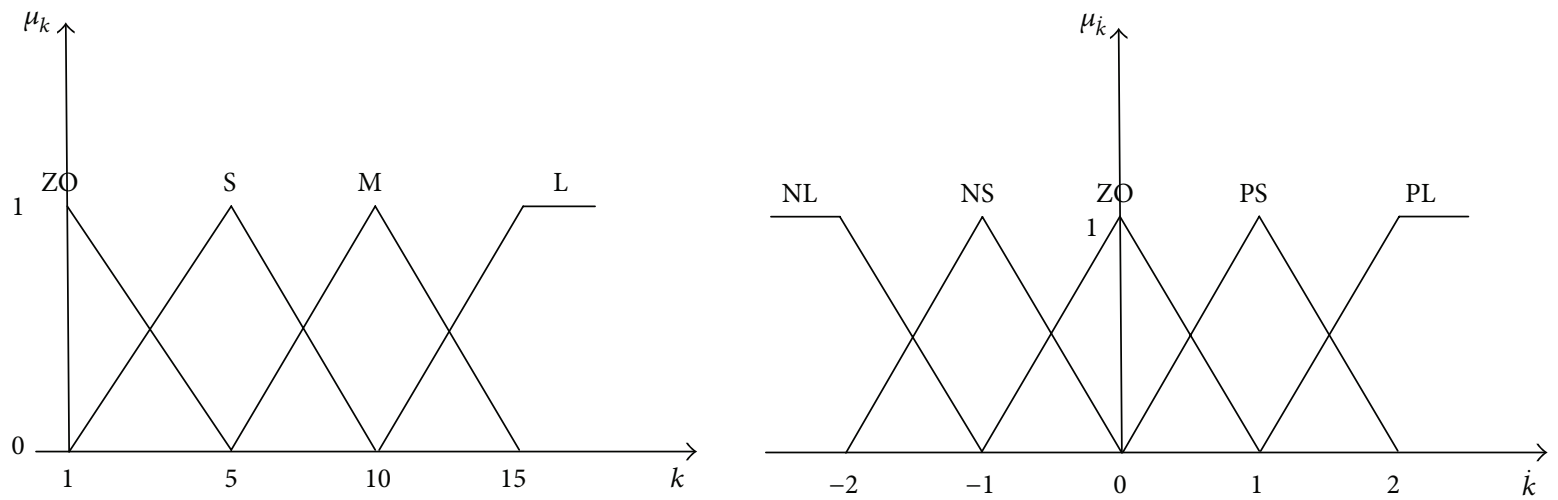

(a)

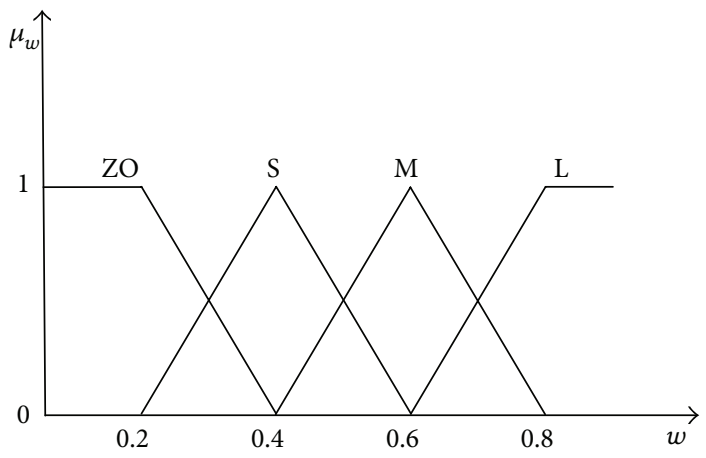

(b)

FIGURE 11: Membership functions for fuzzy reasoning. (a) Input member functions. (b) Output member functions.

Take $\alpha_{1}\left(x_{1}\right)=-c_{1} Z_{1}, c_{1}>0$; then

$$
\dot{Z}_{1}=Z_{2}+\alpha_{1}\left(x_{1}\right)=Z_{2}-c_{1} Z_{1}
$$

The Lyapunov function of the first error subsystem is defined as

$$
\begin{aligned}
V_{1} & =\frac{1}{2} Z_{1}^{2}, \\
\dot{V}_{1} & =Z_{1} \dot{Z}_{1}=-c_{1} Z_{1}^{2}+Z_{1} Z_{2}, \\
\dot{Z}_{2} & =\dot{x}_{2}-\dot{\alpha}_{1}\left(x_{1}\right) \\
& =m\left(x_{1}, x_{2}\right)+n u+c_{1} \dot{Z}_{1} \\
& =m\left(x_{1}, x_{2}\right)+n u-c_{1}^{2} Z_{1}+c_{1} Z_{2} .
\end{aligned}
$$

The Lyapunov function of the second error subsystem is defined as

$$
V_{2}=V_{1}+\frac{1}{2} Z_{2}^{2}
$$

$$
\begin{aligned}
\dot{V}_{2} & =\dot{V}_{1}+Z_{2} \dot{Z}_{2} \\
& =-c_{1} Z_{1}^{2}+Z_{1} Z_{2}+Z_{2}\left[m\left(x_{1}, x_{2}\right)+n u+c_{1} \dot{Z}_{1}\right] \\
& =-c_{1} Z_{1}^{2}+Z_{1} Z_{2}+Z_{2}\left[m\left(x_{1}, x_{2}\right)+n u-c_{1}^{2} Z_{1}+c_{1} Z_{2}\right] .
\end{aligned}
$$

Take the control law for $u$ as follows:

$$
u=\frac{1}{n}\left[-m\left(x_{1}, x_{2}\right)-\left(1-c_{1}^{2}\right) Z_{1}-\left(c_{2}+c_{1}\right) Z_{2}\right]
$$

Then

$$
\dot{V}_{2}=-c_{1} Z_{1}^{2}-c_{2} Z_{2}^{2} \leq 0, \quad c_{1}>0, c_{2}>0 .
$$

By the Lyapunov stability theory, the designed control system can reach a steady state in a limited time, so the system has the stability.

In order to grasp objects with different stiffness stably, a stiffness fuzzy observer is designed to adjust $m\left(x_{1}, x_{2}\right)$. The employed fuzzy logic reasoning has double inputs $(k$ and $\dot{k}$ ) 
TABLE 1: Fuzzy reasoning rules.

\begin{tabular}{|c|c|c|c|c|c|}
\hline \multicolumn{2}{|c|}{$w$} & \multicolumn{4}{|c|}{$k$} \\
\hline & & $\mathrm{ZO}$ & S & $\mathrm{M}$ & $\mathrm{L}$ \\
\hline \multirow{5}{*}{$k$} & NL & $\mathrm{ZO}$ & $\mathrm{ZO}$ & $\mathrm{S}$ & $\mathrm{M}$ \\
\hline & NS & $\mathrm{ZO}$ & $S$ & $\mathrm{~S}$ & $\mathrm{M}$ \\
\hline & $\mathrm{ZO}$ & $\mathrm{ZO}$ & S & $\mathrm{M}$ & $\mathrm{L}$ \\
\hline & PS & $S$ & S & $\mathrm{M}$ & $\mathrm{L}$ \\
\hline & PL & $S$ & $\mathrm{M}$ & $\mathrm{L}$ & $\mathrm{L}$ \\
\hline
\end{tabular}

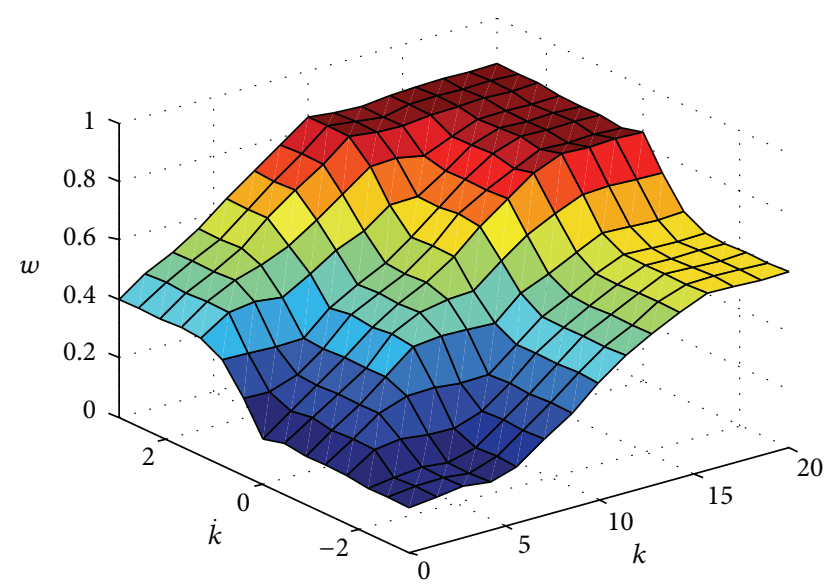

FIGURE 12: Input-output relationship surface map for fuzzy reasoning.

and a single output $(w) . k$ and $\dot{k}$ are, respectively, the stiffness and the stiffness' derivative. $m\left(x_{1}, x_{2}\right)$ is adjusted as follows:

$$
m\left(x_{1}, x_{2}\right)=-(1+w) \frac{B}{J} x_{2}-\frac{D}{J} .
$$

During fuzzification and defuzzification, the inputs and the output types are defined as several fuzzy sets with trigonometry/trapezoidal membership functions as shown in Figure 11. The fuzzy reasoning rules for $w$ are shown in Table 1 . Figure 12 shows the overall input-output relationship of the fuzzy logic reasoning.

3.3. Tactile Representation System. There are lots of neurons distributed on the skin all over the body. The mechanism is called tactile perception. These neurons can receive the information (temperature, humidity, pain, pressure, vibration, etc.) outside the body. One of the most common phenomena of tactile perception is that when natural hand touches an object, the characteristics of the object such as the shape and surface roughness can be felt. Tactile perception is a way to obtain information. It involves human physiology, tactile physiology, tactile phenomenon, and so forth $[10,11]$.

When working in this, tactile representation is important. It is important to transmit grasp force and slide information back to the user.
3.3.1. Grasp Force Detection. In order to achieve a more comprehensive and more accurate measurement of the grasp force, several force sensors are developed [12, 13]. Figure 13(a) shows the thumb of the prosthetic hand. A force sensor (FSS SMT Series, Honeywell) is installed on the tip of the finger; the other two half bridges (Wheatstone half bridge) are separately fixed on the middle and the root of the thumb. This kind of distribution is utilized because the force points on the finger may be different when user grasps objects.

The sensitivity of the force sensor is $12.2 \mathrm{mV} / \mathrm{N}$. The force, $F_{1}$, applied to area 1 is as follows:

$$
F_{1}=\frac{u_{F}}{12.2 \mathrm{mV}},
$$

where $u_{F}$ is the output of the force sensor.

Four strain gauges (sg1, sg2, sg3, and sg4) which have the same properties (material, size, strain coefficient, etc.) are attached to the thumb of the prosthetic hand as shown in Figure 13. $R_{1}, R_{2}, R_{3}$, and $R_{4}$ represent the resistance of these strain gauges, respectively. And $R_{1}, R_{2}, R_{3}$, and $R_{4}$ are of the same value.

The thumb of the prosthetic hand is made of aluminum. There is a deformation in the thumb when the force is applied to it. The deformation of the thumb will lead to the corresponding deformation of the strain gauges. And the deformation of the strain gauges will lead to the change of the resistances' value of these strain gauges.

Take sg1 and sg2; for example, tensile deformation and compressive deformation occurred in sg1 and sg2, respectively, when the force is applied to the thumb. Tensile deformation of sg1 results in the increasing of the resistance' value of sg1. On the contrary, compressive deformation of sg2 results in the decreasing of the resistance' value of sg2. The variations of the resistance' value of sg1 and sg2 are considered to be the same for the reason that the deformation is small and sg1 and sg2 have the same properties.

sg1 and sg2 are connected in the measurement circuit as shown in Figure 13(b). sg1, sg2, and two additional resistances $\left(R_{a 1}, R_{a 2}\right)$ constitute a Wheatstone bridge. The output of the circuit, $U_{g 1}$, is as follows:

$$
\begin{aligned}
U_{g 1} & =\frac{R_{2}-\Delta R}{\left(R_{1}+\Delta R\right)+\left(R_{2}-\Delta R\right)} \times E-\frac{R}{R+R} \times E \\
& =-\frac{\Delta R}{2 \times R_{1}} \times E
\end{aligned}
$$

where $E$ is the power voltage supplied to the circuit, $\Delta R$ is the change of resistance of sgl and $\mathrm{sg} 2$, and $R$ is the value of $R_{a 1}$ and $R_{a 2}$.

Because the force, $F_{2}$, applied to area 2 (as shown in Figure 13) is proportional to the deformation of the strain gauges, it can be obtained by measuring the output of the circuit $\left(U_{g 1}\right.$, shown in Figure 13(b)).

The force, $F_{3}$, applied to area 3 can be obtained by using the same method.

When the prosthetic hand contacts the object in areas 1 , 2 , and 3, the grasp forces are measured by force sensor, half bridge 1 , and half bridge 2 , respectively. 


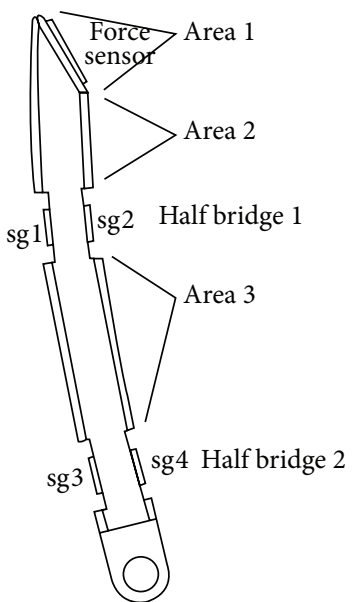

(a)

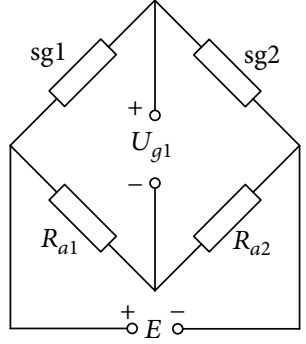

(b)
FIGURE 13: The thumb of the prosthetic hand and the sensor measure circuit.

3.3.2. Sliding State Detection. A polyvinylidene fluoride (PVDF) piezoelectric film is often used for tactile sensor design to detect the sliding for its excellent dynamic characteristics and physical characteristics, such as light quality, soft, large contact resistance, and plasticity $[14,15]$. In this paper, a PVDF piezoelectric film is attached to the surface of the silicone glove of the prosthetic hand. The signal in Figure 14(a) shows a sliding during a grasp operation. In this figure, $T$ indicates the time span of a sliding process.

To reduce the influence of noise on sliding detection, the filtering process is implemented. In addition, a small threshold is subtracted. Figure 14(b) shows the sliding signals after these two processes. Compared with the sliding signals, temperature is a slow change variable. The influence of the temperature is ignored when using PVDF to detect the slide state.

According to the signals shown in Figure 14 and the characteristics of the PVDF, the number of zero-crossing per time unit is adopted to indicate the sliding situation.

3.3.3. Tactile Representation. The main tactile representation techniques are pneumatic stimulation, vibration stimulation, functional neuromuscular stimulation, thimble stimulation, thermal stimulation, and so forth [16]. The vibration stimulation is adopted because it is convenient to use and does not cause damage to human body. The vibration coding patterns, including vibration frequency, amplitude, duration, rhythm, and order [17, 18], affect the accuracy of the tactile perception of the user directly. However, due to the existence of the tactile illusion phenomenon, an efficient vibration coding pattern must be established. The tactile illusion is a kind of phenomenon in which tactile perceptions do not match the objective stimulation. Many kinds of tactile illusion phenomena have already been discovered, such as phantom sensation and apparent movement. The reasonable utilization of these phenomena may contribute to realization of the tactile representation.

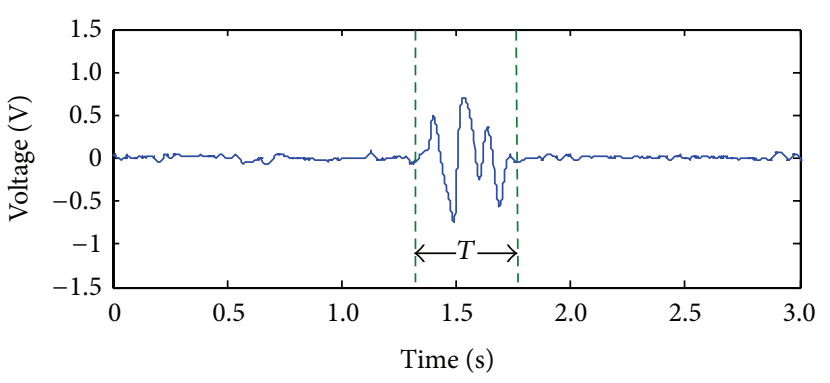

(a)

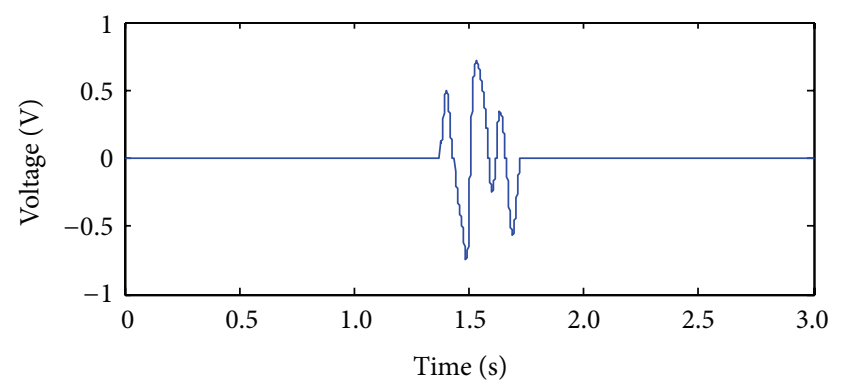

(b)

FIGURE 14: Sliding signals.

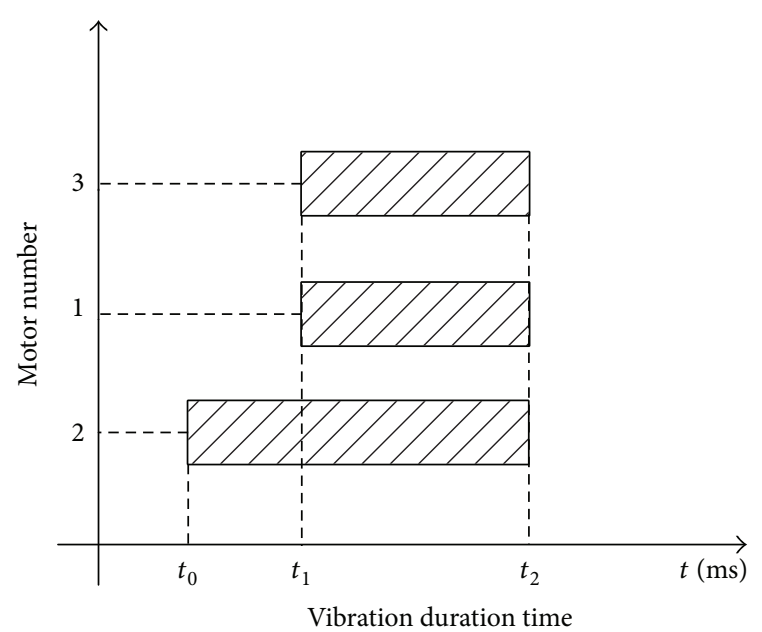

FIGURE 15: The coding pattern of the grasp force tactile representation.

A coding pattern (vibration coding) of grasp force is designed. As shown in Figure 15, when the grasp force is detected, motor number 2 begins to vibrate, and then motor number 1 and motor number 3 begin to vibrate. $t_{0}$ is the beginning time of motor number $2, t_{1}$ is the beginning time of motors number 1 and number 3 , and $t_{2}$ is the ending time of all these motors. $t_{0}$ is the time of grasp force being detected as well, and $t_{2}$ shows the time when the grasp force reduces to zero. The interval between $t_{0}$ and $t_{1}$ is $300 \mathrm{~ms}$. The grasp force measured by the sensor is used to modulate the vibration strength (VS) according to the principle as shown in formula 


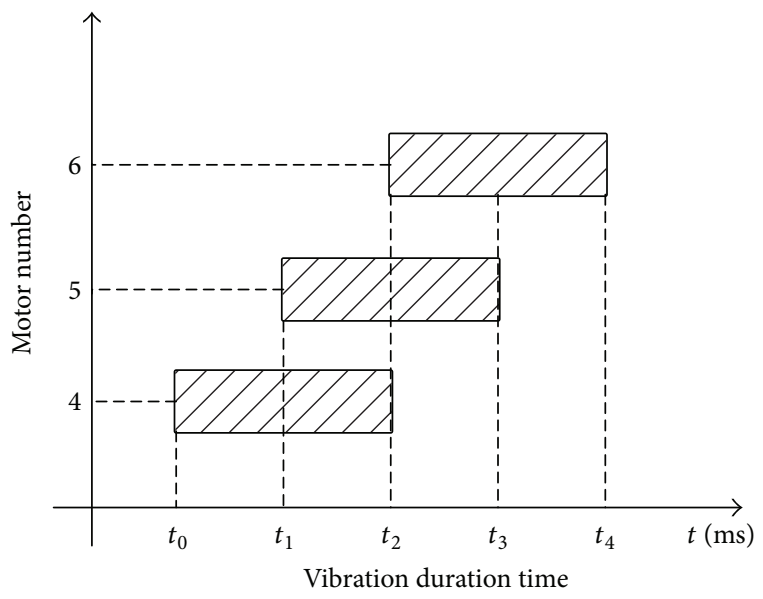

Figure 16: The coding pattern of the sliding tactile representation.

TABLE 2: Relationship between vibration strength level and voltage applied to motor.

\begin{tabular}{lc}
\hline Vibration strength level & Voltage applied to motor (V) \\
\hline I & 1.0 \\
II & 2.0 \\
III & 3.0 \\
IV & 4.0 \\
V & 5.0 \\
\hline
\end{tabular}

(19), and the vibration frequency is set to $100 \mathrm{~Hz}$. By this method, the user may feel his/her arm being grasped:

$$
\text { VS }= \begin{cases}\text { I } & 0<r \leq 0.2 \\ \text { II } & 0.2<r \leq 0.4 \\ \text { III } & 0.4<r \leq 0.6 \\ \text { IV } & 0.6<r \leq 0.8 \\ \text { V } & 0.8<r \leq 1.0\end{cases}
$$

where I, II, III, IV, and V are the vibration strength levels; the relationship between vibration strength levels and the voltage applied to the motor is as shown in Table 2. $r$ is the ratio of grasp force detected by the force sensors to the maximum allowable value of the grasp force:

$$
r=\frac{F}{F_{\max }},
$$

where $F$ is the grasp force detected by the force sensors and $F_{\text {max }}$ is the maximum allowable value of the grasp force.

A sliding tactile representation coding pattern is designed according to the phenomenon of apparent movement (see appendix) [19]. The vibration strength is set to level III, and the vibration frequency is set to $100 \mathrm{~Hz}$. As shown in Figure $16, t_{0}, t_{1}$, and $t_{2}$ are, respectively, the beginning time of the motors number 4 , number 5 , and number 6 . The ending times of these three motors, respectively, are $t_{2}, t_{3}$, and $t_{4}$. The time intervals of $t_{0} \sim t_{1}, t_{1} \sim t_{2}, t_{2} \sim t_{3}$, and $t_{3} \sim t_{4}$ are

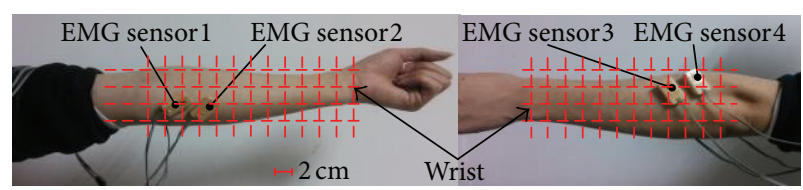

FIgURE 17: Laminating position of the EMG sensors.

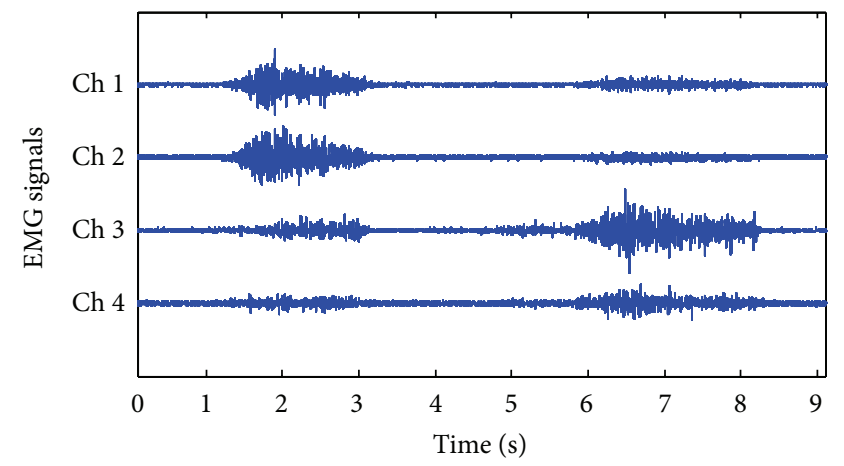

FIGURE 18: EMG signals coming from 4 EMG sensors.

TABLE 3: Combination of the EMG signals.

\begin{tabular}{ll}
\hline Group & Composition \\
\hline 1 & Ch1 and Ch3 \\
2 & Ch1 and Ch4 \\
3 & Ch2 and Ch3 \\
4 & Ch2 and Ch4 \\
\hline
\end{tabular}

$250 \mathrm{~ms} . t_{0}$ is the time of sliding situation being detected as well. By this method, the user may feel something sliding on his/her arm.

\section{Experiments and Results}

To verify the validity of the control strategy presented in this paper, lab-based experiments were carried out.

4.1. Evaluation Experiments of the EMG Self-Learning Motion Recognition Method. Motion recognition experiment was conducted to verify the effectiveness of the EMG self-learning motion recognition method. In the experiment, four EMG sensors were distributed on the participants' forearms as shown in Figure 17. The participants executed hand motions (hand grasp and hand open) according to the commands. Figure 18 shows the EMG signals when participants executed the hand motions.

According to the laminating positions of the EMG sensors, EMG signals coming from four sensors can be combined into four groups as shown in Table 3. Two recognition methods, including one shown in Figure 1 and the other EMG self-learning recognition method, have been applied to these four groups EMG signals. Figure 19 shows the recognition results by using the recognition method as shown in Figure 1, and Figure 20 shows the recognition results by using the EMG self-learning recognition method. 
TABle 4: Correlation coefficients between every two recognition results by using the recognition method shown in Figure 1.

\begin{tabular}{lcccc}
\hline Correlation coefficient (\%) & $\begin{array}{c}\text { Recognition results } \\
\text { of group 1 }\end{array}$ & $\begin{array}{c}\text { Recognition results } \\
\text { of group 2 }\end{array}$ & $\begin{array}{c}\text { Recognition results } \\
\text { of group 3 }\end{array}$ & $\begin{array}{c}\text { Recognition results } \\
\text { of group 4 }\end{array}$ \\
\hline Recognition results of group 1 & 100 & 89.39 & 94.35 & 87.84 \\
Recognition results of group 2 & & 100 & 90.49 & 97.91 \\
Recognition results of group 3 & & & 100 & 91.58 \\
Recognition results of group 4 & & & & 100 \\
\hline
\end{tabular}

TABLE 5: Correlation coefficients between every two recognition results by using the EMG self-learning recognition method.

\begin{tabular}{lcccc}
\hline Correlation coefficient (\%) & $\begin{array}{c}\text { Recognition results } \\
\text { of group 1 }\end{array}$ & $\begin{array}{c}\text { Recognition results } \\
\text { of group 2 }\end{array}$ & $\begin{array}{c}\text { Recognition results } \\
\text { of group 3 }\end{array}$ & $\begin{array}{c}\text { Recognition results } \\
\text { of group 4 }\end{array}$ \\
\hline Recognition results of group 1 & 100 & 99.20 & 99.25 & 98.11 \\
Recognition results of group 2 & & 100 & 98.28 & 99.53 \\
Recognition results of group 3 & & & 100 & 99.04 \\
Recognition results of group 4 & & & & 100 \\
\hline
\end{tabular}

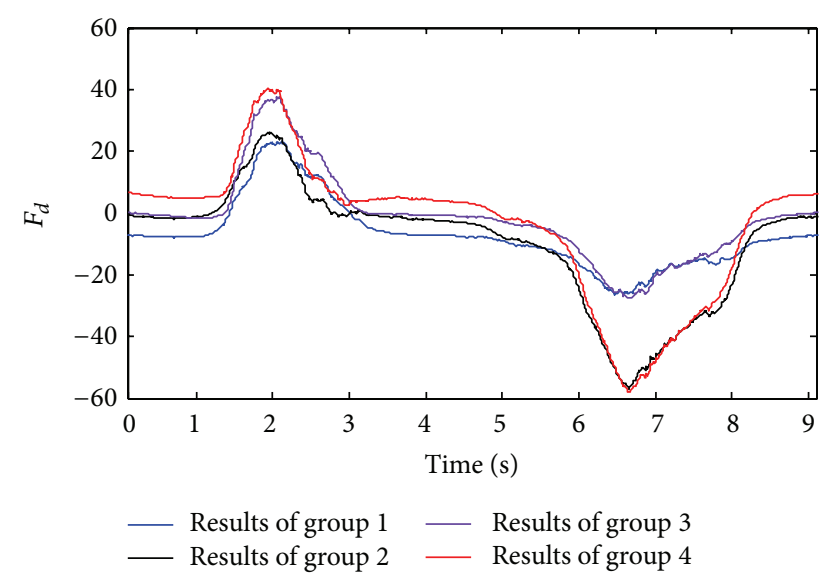

FIGURE 19: Motion recognition results by using the recognition method shown in Figure 1.

To evaluate the consistency of the recognition results, the correlation coefficients between every two recognition results were calculated as shown in Tables 4 and 5. Correlation coefficient $(\rho)$ is a measurement of the linear correlation (dependence) between two variables $X$ and $Y$. It is widely used as a measurement of the degree of linear dependence between two variables. The formula for $\rho$ is

$$
\rho_{X, Y}=\frac{\operatorname{cov}(X, Y)}{\sigma_{X} \sigma_{Y}}=\frac{E\left[\left(X-\mu_{X}\right)\left(Y-\mu_{Y}\right)\right]}{\sigma_{X} \sigma_{Y}},
$$

where cov is the covariance, $\sigma_{X}$ is the standard deviation of $X, \mu_{X}$ is the mean of $X$, and $E$ is the expectation.

From the results of the EMG motion recognition experiment, recognition results by using the EMG self-learning recognition method have a better consistency. EMG selflearning can reduce the influence on EMG signals caused by the uncertainty of the contacting position of the EMG sensors.

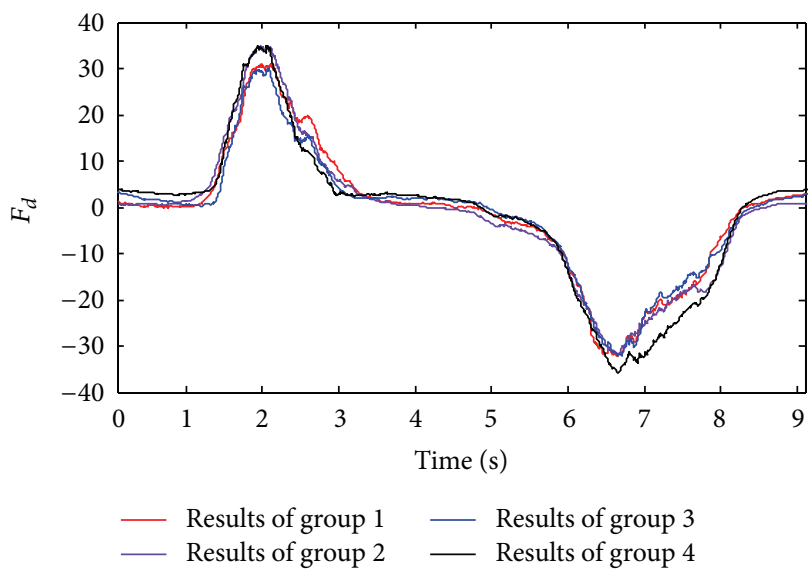

FIGURE 20: Motion recognition results by using the EMG selflearning recognition method.

4.2. Evaluation Experiments of the Backstepping Controller. To verify the effectiveness of the BCSFO, velocity tracking and force tracking experiments were implemented. The designed speed was inputted to the controller in free space, while the designed grasp force was inputted to the controller in restricted space. The results are shown in Figures 21 and 22. The objects with different stiffness were grasped by prosthetic hand in the experiment, and the results are shown in Table 6. Table 6 contains the mean value $(\bar{e})$ and mean variance $(R)$ of the force tracking error. The results show that the controller can track the designed velocity and designed grasp force quickly; the tracking error is in the acceptable range.

4.3. Tactile Representation Coding Experiment and Results. Ten nonamputee volunteers (five males and five females, aged from 22 to 27) were chosen to use the MPH-III. The EMG acquisition device was worn on the forearm, and the EMG sensors were put on a pair of antagonistic muscles. The TRD was worn on the upper arm. Five minutes or more was given 

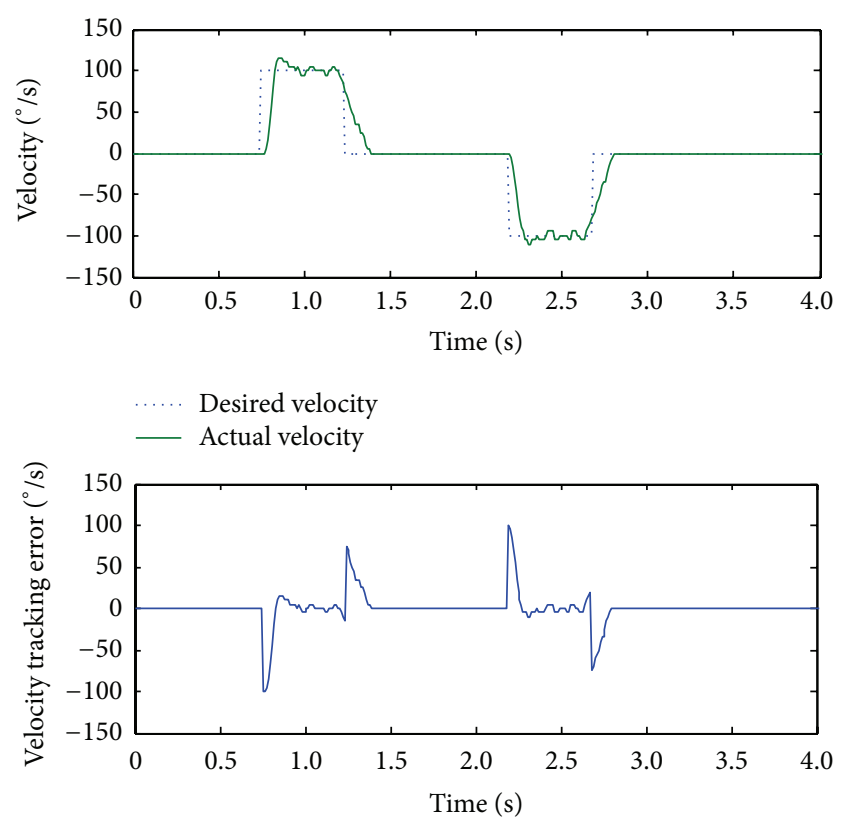

(a)

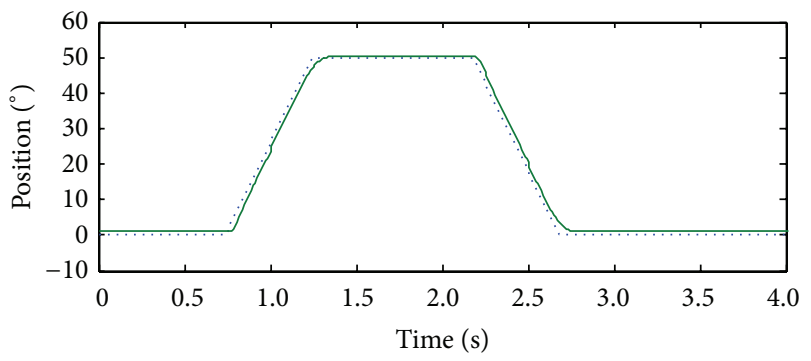

Desired velocity

- Actual velocity

(b)

FIGURE 21: Results of the velocity tracking experiment. (a) Velocity tracking curve and tracking error curve. (b) The expected position and actual position.

to each participant to get familiar with the prosthetic hand. After experiments, they graded these three control strategies.

The TRD outputs the grasp force vibration coding for 25 times. Among them, the sliding state vibration coding occurs for five times randomly. The participants were asked to record the vibration state according to their feelings. Each vibration state occurs 5 times. Each participant accomplished experiments without influence from others. The results of the experiment are shown in Figures 23 and 24.

In Figure 23, $x$-axis represents the actual strength level outputted by the TRD; $y$-axis represents the times the participants recorded the strength level. The different strength levels the participant felt are represented by different colors. That is to say, the strength levels I, II, III, IV, and V the participants felt are represented by red, yellow, blue, green, and black, respectively. Red, yellow, blue, green, and black represent the strength levels the participator felt which are I, II, III, IV, and $\mathrm{V}$, respectively.

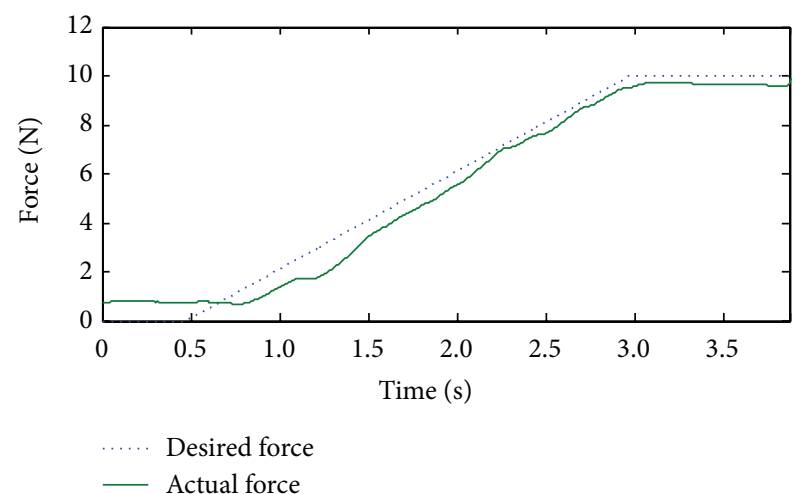

(a)

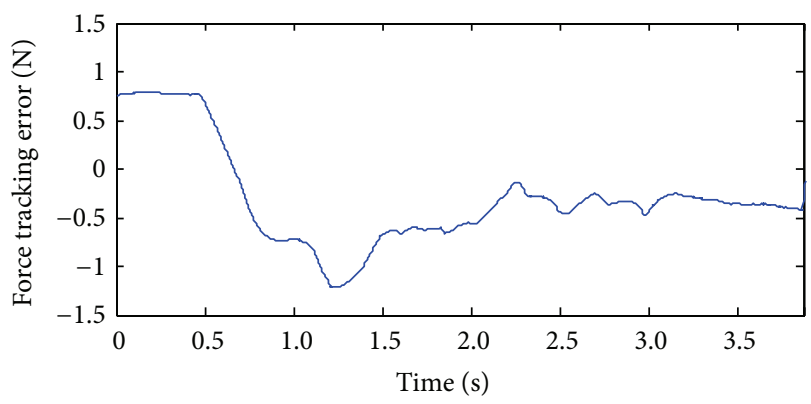

(b)

FIGURE 22: Force tracking curve and tracking error curve.

TABLE 6: Grasp results of different objects.

\begin{tabular}{lccc}
\hline Object's number & Object's stiffness $(\mathrm{N} / \mathrm{mm})$ & $\bar{e}(\mathrm{~N})$ & $R(\mathrm{~N})$ \\
\hline 1 & 0.5 & 0.82 & 1.00 \\
2 & 1 & 1.46 & 1.33 \\
3 & 2 & 0.88 & 1.02 \\
4 & 4 & 1.43 & 1.27 \\
5 & 10 & 1.22 & 1.10 \\
\hline
\end{tabular}

Take the experimental results of participant 1; for example, when the actual strength level is "I" (see $x$-axis), the times of the strength levels I, II, III, IV, and V perceived by the participants are 5, 0, 0, 0 , and 0 , respectively. When the actual strength level is "II," the times of the strength levels I, II, III, IV, and V perceived by the participants are $0,5,0,0$, and 0 , respectively. When the actual strength level is "III," the times of the strength levels I, II, III, IV, and V perceived by the participants are $0,0,4,1$, and 0 , respectively. When the actual strength level is "IV," the times of the strength levels I, II, III, IV, and V perceived by the participants are $0,0,1,3$, and 1 , respectively. When the actual strength level is " $V$," the times of the strength levels I, II, III, IV, and V perceived by the participants are $0,0,0,1$, and 4 , respectively.

Figure 24 shows the average values of each strength level perceived by the participants in each actual strength level.

The results of Figures 23 and 24 show that the participants can distinguish the strength level precisely when the strength level of tests is given in low level (levels 1 and 2), while on the condition of high level (levels 3 to 5), the strength 

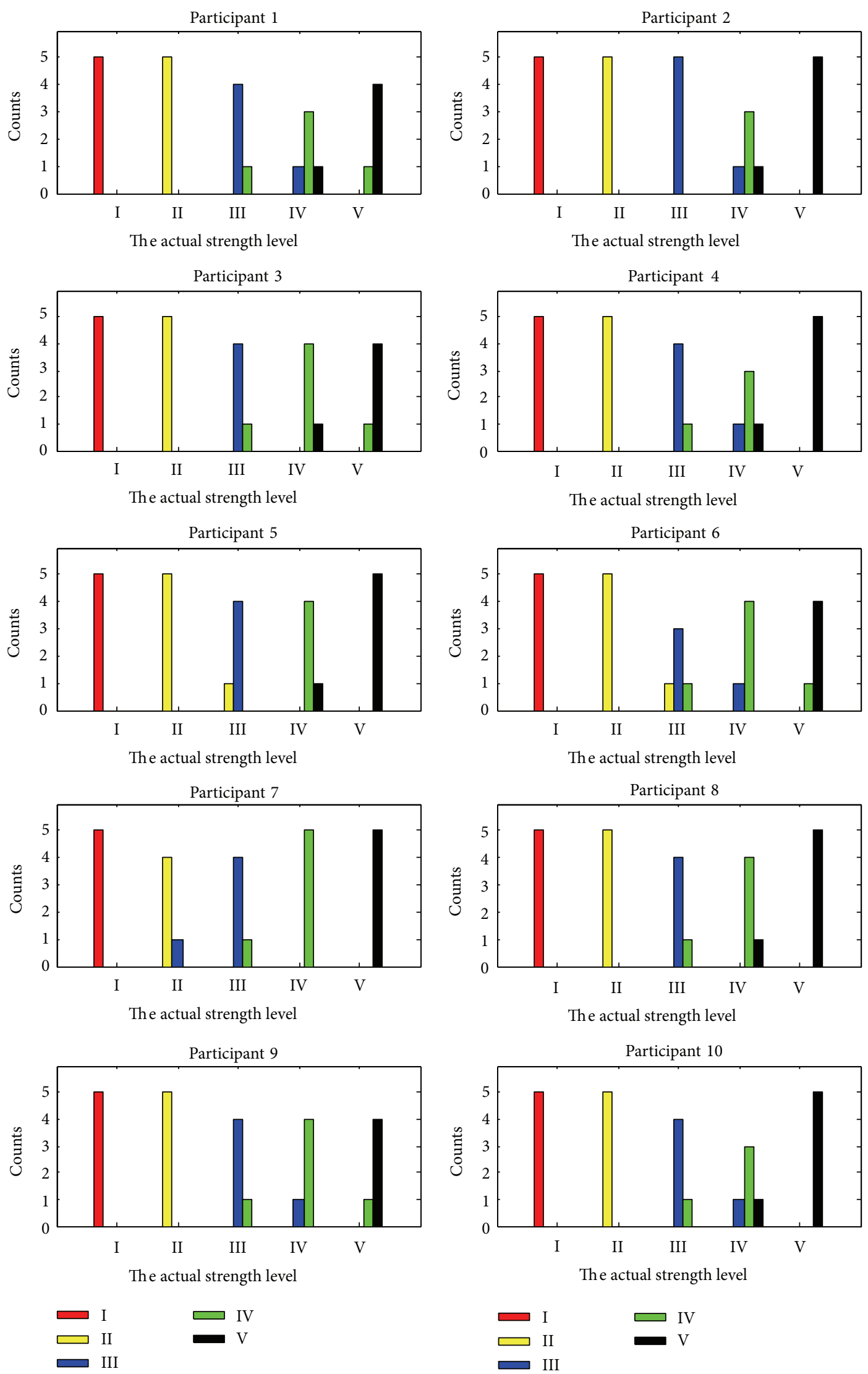

Figure 23: Results of the grasp force coding experiment. 


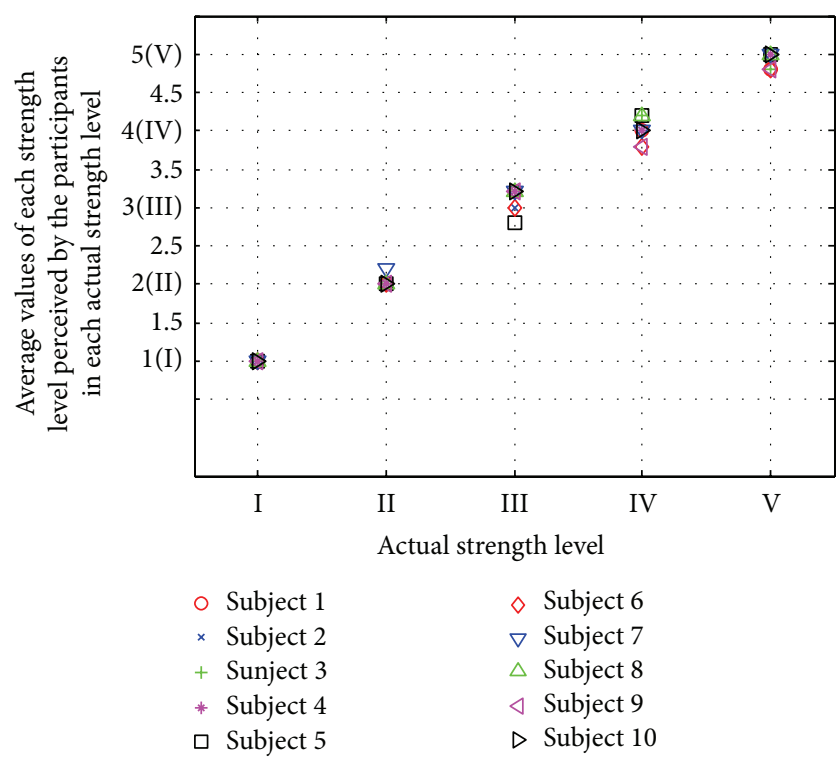

Figure 24: Average values of each strength level perceived by the participants in each actual strength level.

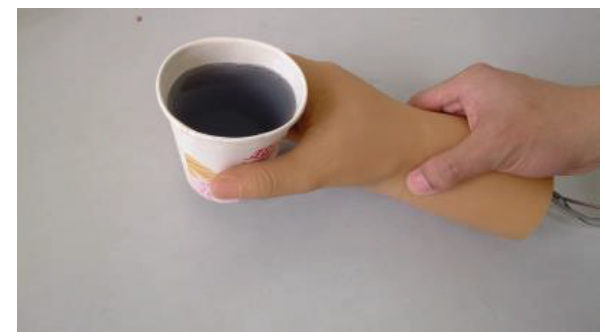

FIGURE 25: Experiment scene of grasping.

level perceived by the participants in each actual strength level fluctuates around the actual strength level. The errors between false records and correct records are no more than 1 level. The results of all the participants show the same trend. In the view of the experimental results, this kind of vibration coding can help users to perceive the grasp force of the prosthetic hand.

The experimental result of tactile representation of sliding state is as its expected because the coding pattern is simple and there is no relationship between the siding coding pattern and grasp force tactile feedback. Most of the participants can make the judgment whether the sliding occurs or not.

4.4. Grasp Experiments and Results. The force control strategy (M1) shown in Figure 1, the EMG self-learning control strategy (M2), and control strategy shown in Figure 2 (M3) were compared with each other. These three strategies all contain visual feedback. The differences are that M2 has EMG self-learning function and the controller in M2 is BCSFO. Besides the features of M2, M3 has an additional function of tactile feedback. The experiments assume that the functions of visual feedback in these three strategies are the same. Five minutes was given for participants to get familiar with

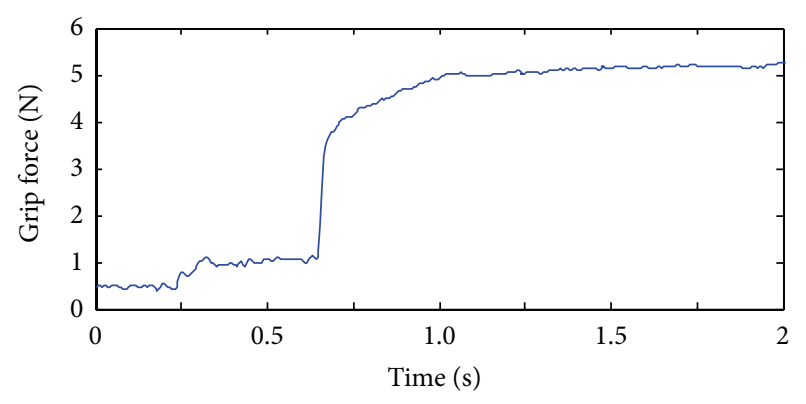

FIGURE 26: Grasp force curve.

these control strategies. Then, ten participants were asked to grasp and lift a paper cup which was full of water (see Figure 25). In this process, the participant should keep water without overflowing or dropping. All control strategies were tested for ten times. The number of successful lifting was used to quantify the performance of the control strategy. And the participants were asked to evaluate every control strategy after experiments according to their feeling about the performance of the control strategy. And the performance includes flexibility and usability. The best control strategy was marked as highest grade, 10. Table 7 shows the results of the experiment. Figure 26 shows the grasp force curve which was recorded in the process of a participant grasping a paper cup by using the control strategy of M3.

In this experiment, the comments from all participants show that the M3 is the best one among these three strategies. When using prosthetic hand without EMG self-learning, most of the participants have to adjust the positions of the EMG sensors on the skin surface, while it is unnecessary to adjust by using the strategies with EMG self-learning.

\section{Conclusions}

The control strategy with tactile feedback for EMG prosthetic hand is described in detail. Aiming at reducing the influence on the EMG signals which comes from the attaching positions of EMG sensors, an EMG self-learning recognition method is proposed. A BCSFO is proposed to realize the velocity proportional control in free space and grasp force tracking control in restricted space. A tactile representation system is designed to help the user perceive the state of the prosthetic hand, and the states include grasp force and slid information.

The experiments are implemented to verify the effect of the proposed control strategy. And the results show that the different contacting positions between sensors and arm lead to the variance of the EMG signals, and this kind of influence can be reduced by the proposed EMG self-learning method. The proposed BCSFO can meet the requirements of the prosthetic hand (velocity proportional control in free space and grasp force tracking control in restricted space). And the results of the grasping experiments show that the strategy with EMG self-learning method and tactile feedback (M3) is better than the strategy of the force control (M1) and 
TABLE 7: Results of the paper cup grasping experiment.

\begin{tabular}{lccc}
\hline & $\begin{array}{c}\text { Mean success rate of paper } \\
\text { cup grasping (\%) }\end{array}$ & $\begin{array}{c}\text { Standard deviation of paper } \\
\text { cup grasping (\%) }\end{array}$ & $\begin{array}{c}\text { Mean score marked by the } \\
\text { test participants }\end{array}$ \\
\hline Force control (M1) & 51 & 18 & 6.35 \\
EMG self-learning force control (M2) & 59 & 20 & 7.25 \\
$\begin{array}{l}\text { EMG self-learning force control with } \\
\text { tactile feedback (M3) }\end{array}$ & 79 & 15 & 10 \\
\hline
\end{tabular}
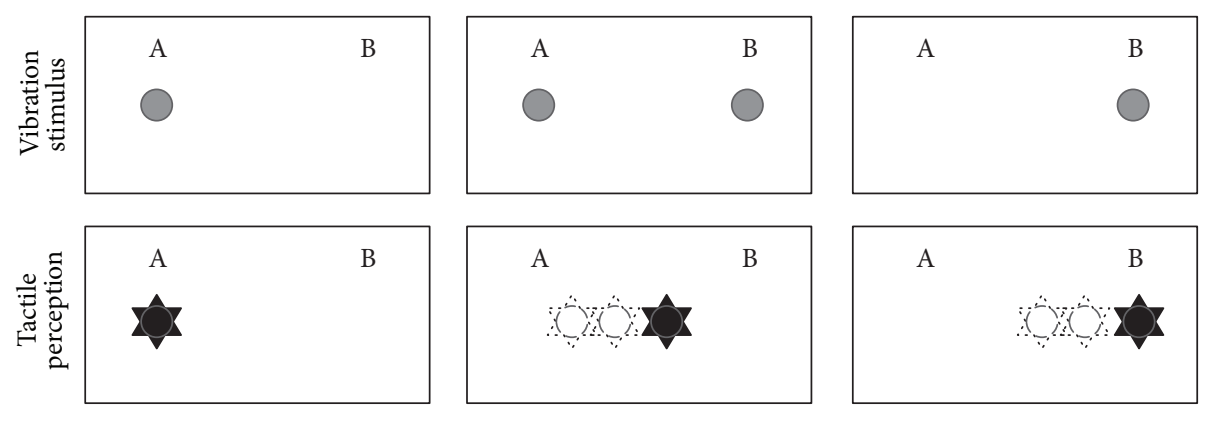

FIGURE 27: Diagram of the apparent movement.

the strategy of the EMG self-learning control (M2) in the aspect of control effect of the prosthetic hand.

Moreover, all the participants think that the EMG signal self-learning pattern recognition method is much more helpful and convenient in the process of manipulating the prosthetic hand.

For the future work, after lots of experiments we will research a more effective coding pattern for tactile representation, which would be easily accepted by amputees.

\section{Appendix}

This phenomenon was described in detail in [19]. Its basic principle is as follows.

(1) Point A starts to vibrate.

(2) Point B starts to vibrate.

(3) Point A stops.

By applying vibration stimulation in this order, the participant will get an illusion that point $\mathrm{A}$ is moving towards point B (see Figure 27).

\section{Conflict of Interests}

The authors declare that there is no conflict of interests regarding the publication of this paper.

\section{Acknowledgment}

This paper was supported by the Nature Science foundation of China under Grant no. 61325018.

\section{References}

[1] H. H. Sears and J. Shaperman, "Proportional myoelectric hand control: an evaluation," American Journal of Physical Medicine and Rehabilitation, vol. 70, no. 1, pp. 20-28, 1991.

[2] M. H. M. Zaini and S. A. Ahmad, "Surgical and non-surgical prosthetic hands control: a review," in Proceedings of the IEEE Symposium on Industrial Electronics and Applications (ISIEA '11), pp. 634-637, 2011.

[3] D. Graupe and W. K. Cline, "Functional separation of EMG signals via ARMA identification methods for prosthesis control purposes," IEEE Transactions on Systems, Man and Cybernetics, vol. 5, no. 2, pp. 252-259, 1975.

[4] T. Khoshaba, K. Badie, and R. M. Hashemi, "EMG pattern classification based on back propagation neural network for prosthesis control," in Proceedings of the 12th Annual International Conference of the IEEE Engineering in Medicine and Biology Society, pp. 1474-1475, November 1990.

[5] K. Mahaphonchaikul, D. Sueaseenak, C. Pintavirooj, M. Sangworasil, and S. Tungjitkusolmun, "EMG signal feature extraction based on Wavelet transform," in Proceedings of the 7th Annual International Conference on Electrical Engineering/Electronics, Computer, Telecommunications and Information Technology (ECTI-CON '10), pp. 327-331, May 2010.

[6] A. Fassih, D. S. Naidu, S. Chiu, and M. P. Schoen, "Precision grasping of a prosthetic hand based on virtual spring damper hypothesis," in Proceedings of the 5th Cairo International Biomedical Engineering Conference (CIBEC '10), pp. 79-82, Cairo, Egypt, December 2010.

[7] C.-H. Chen, D. S. Naidu, A. Perez-Gracia, and M. P. Schoen, "A hybrid adaptive control strategy for a smart prosthetic hand," in Proceedings of the 31st Annual International Conference of the IEEE Engineering in Medicine and Biology Society (EMBC '09), pp. 5056-5059, IEEE, September 2009.

[8] E. D. Engeberg and S. G. Meek, "Backstepping and sliding mode control hybridized for a prosthetic hand," IEEE Transactions on 
Neural Systems and Rehabilitation Engineering, vol. 17, no. 1, pp. 70-79, 2009.

[9] C. Wu, A. Song, and H. Zhang, "Adaptive fuzzy control method for EMG prosthetic hand," Chinese Journal of Scientific Instrument, vol. 34, no. 6, pp. 1339-1345, 2013.

[10] J. Liu and A. Song, "Discrimination and memory experiments on haptic perception of softness," Perceptual and Motor Skills, vol. 106, no. 1, pp. 295-306, 2008.

[11] C. Antfolk, M. D’alonzo, B. Rosén, G. Lundborg, F. Sebelius, and C. Cipriani, "Sensory feedback in upper limb prosthetics," Expert Review of Medical Devices, vol. 10, no. 1, pp. 45-54, 2013.

[12] A. Song, J. Wu, G. Qin, and W. Huang, "A novel self-decoupled four degree-of-freedom wrist force/torque sensor," Measurement, vol. 40, no. 9-10, pp. 883-891, 2007.

[13] J. Ma, A. Song, and D. Pan, "Dynamic compensation for twoaxis robot wrist force sensors," Journal of Sensors, vol. 2013, Article ID 357396, 5 pages, 2013.

[14] A. Song, Y. Han, H. Hu, and J. Li, "A novel texture sensor for fabric texture measurement and classification," IEEE Transactions on Instrumentation and Measurement, vol. 63, no. 7, pp. 17391747, 2013.

[15] H. Hu, Y. Han, A. Song, S. Chen, C. Wang, and Z. Wang, "A finger-shaped tactile sensor for fabric surfaces evaluation by 2-dimensional active sliding touch," Sensors, vol. 14, no. 3, pp. 1588-1592, 2014.

[16] D. Wood, “Tactile displays: present and future," Displays, vol. 18, pp. 125-128, 1998.

[17] S. A. Brewster and L. M. Brown, "Tactons: structured tactile messages for non-visual information display," in Proceedings of the 5th Australasian User Interface Conference (AUIC '04), pp. 15-23, Dunedin, New Zealand, 2004.

[18] K. MacLean and M. Enriquez, "Perceptual design of haptic icons," in Proceedings of Eurohaptics, pp. 351-363, 2003.

[19] G. V. Bekesy, "Sensation on the skin similar to directional hearing, beats, and harmonics of the ear," Journal of the Acoustic Society of America, vol. 29, no. 4, pp. 489-501, 1957. 

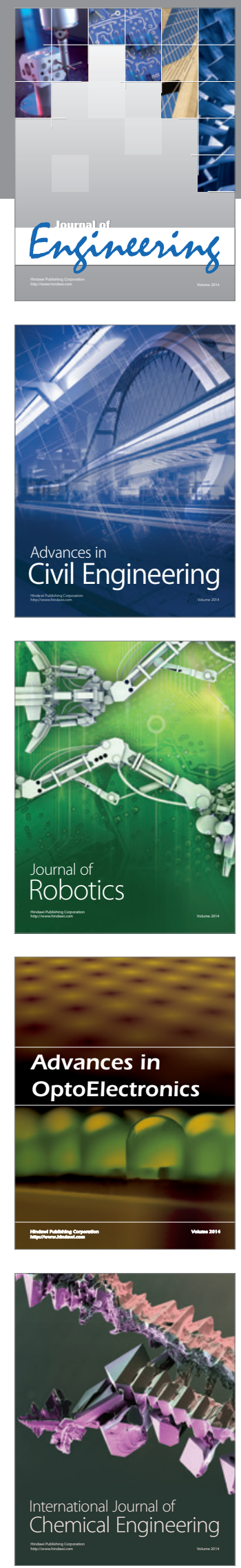

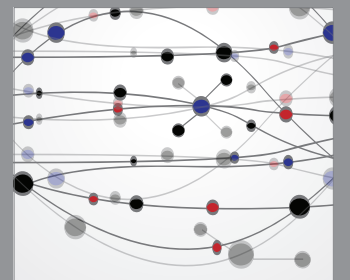

The Scientific World Journal
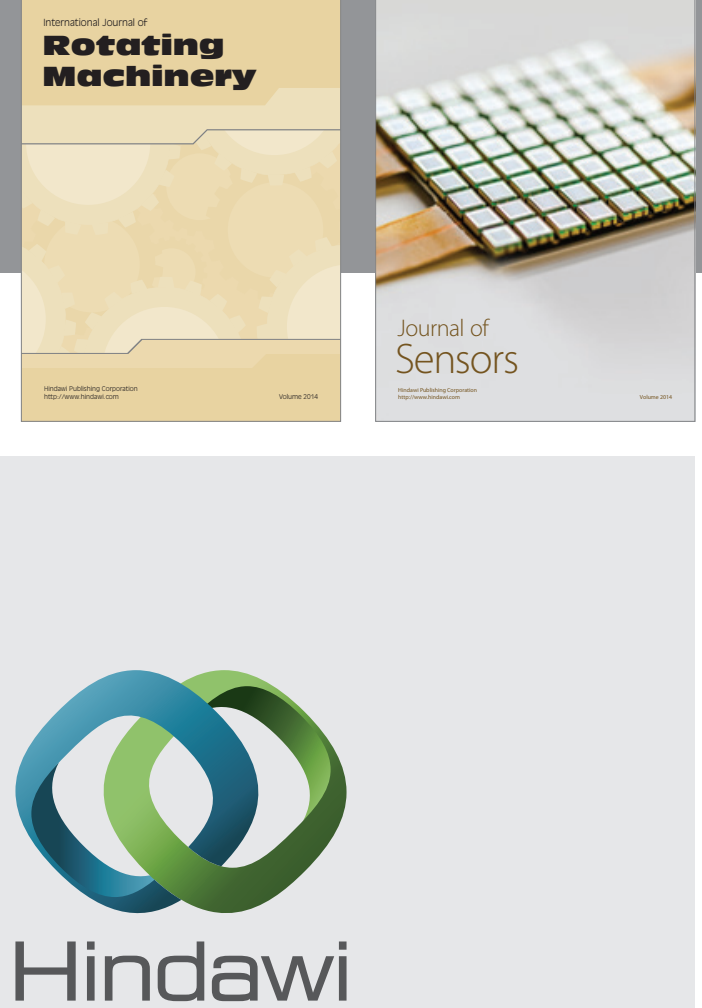

Submit your manuscripts at http://www.hindawi.com
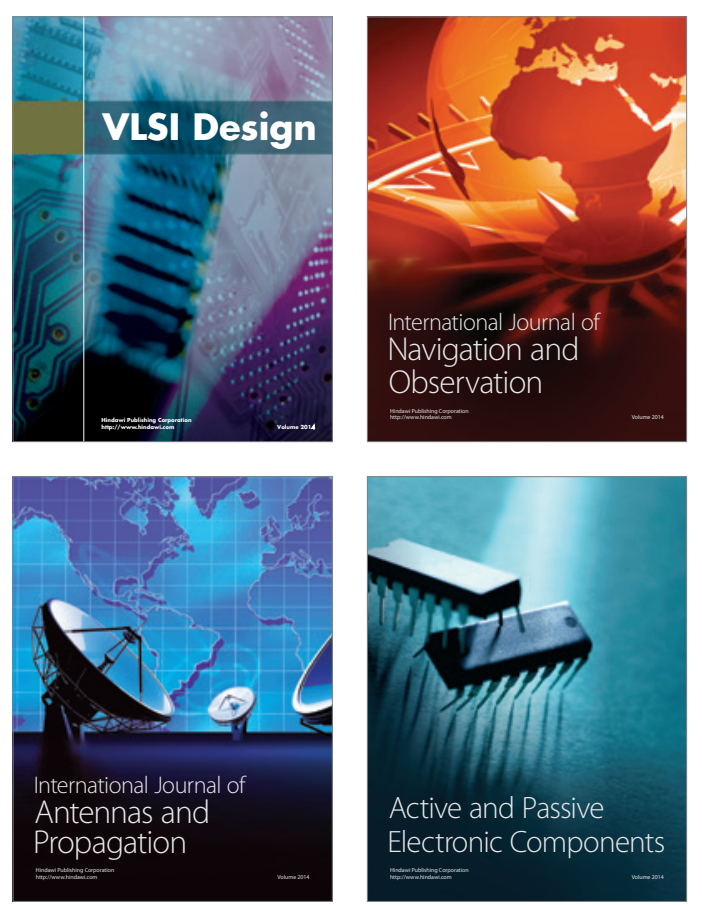
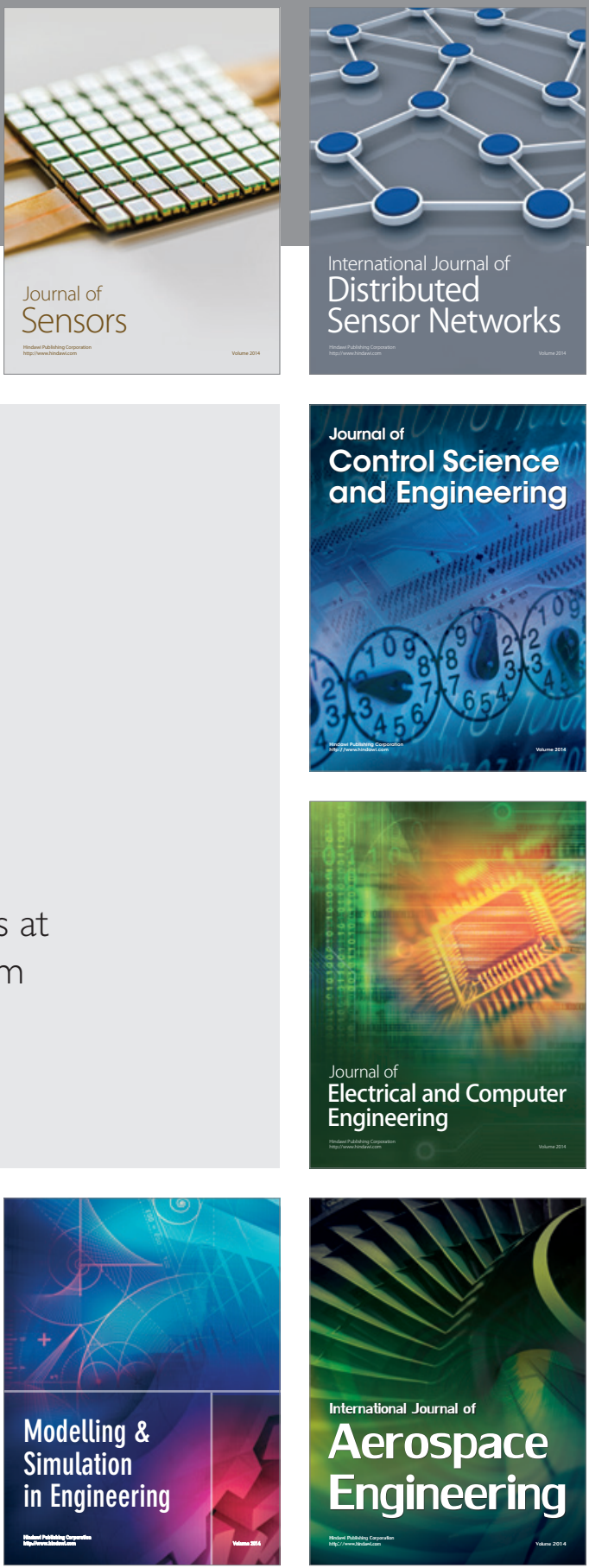

Journal of

Control Science

and Engineering
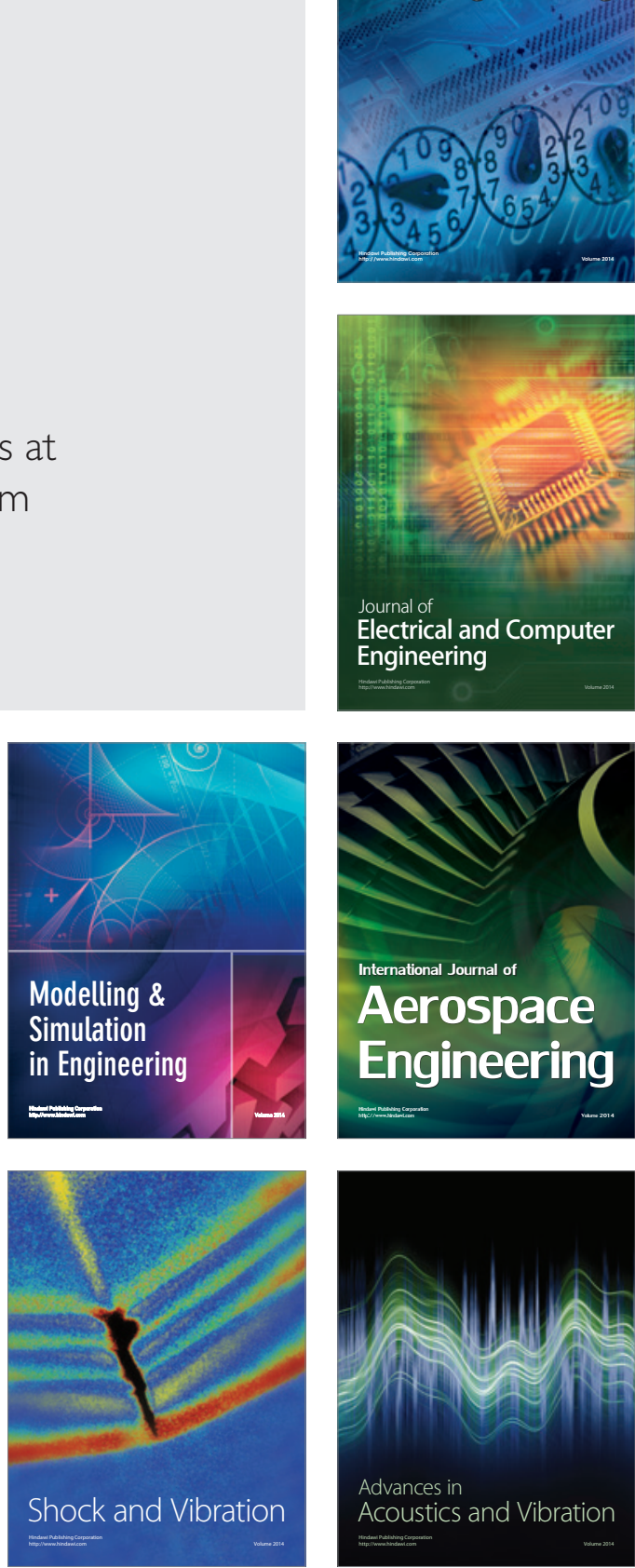